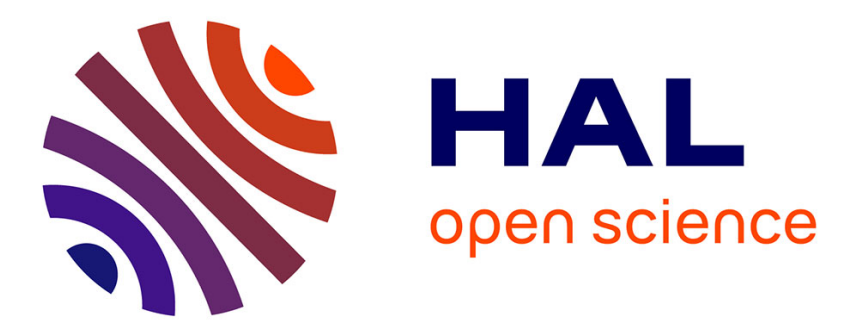

\title{
Active joint visco-elasticity estimation of the human knee using FES
}

Seiya Sakaguchi, Gentiane Venture, Christine Azevedo Coste, Mitsuhiro

Hayashibe

\section{- To cite this version:}

Seiya Sakaguchi, Gentiane Venture, Christine Azevedo Coste, Mitsuhiro Hayashibe. Active joint viscoelasticity estimation of the human knee using FES. BioRob: Biomedical Robotics and Biomechatronics, Jun 2012, Roma, Italy. pp.1644-1649, 10.1109/BioRob.2012.6290727 . lirmm-00736801

\section{HAL Id: lirmm-00736801 https://hal-lirmm.ccsd.cnrs.fr/lirmm-00736801}

Submitted on 30 Sep 2012

HAL is a multi-disciplinary open access archive for the deposit and dissemination of scientific research documents, whether they are published or not. The documents may come from teaching and research institutions in France or abroad, or from public or private research centers.
L'archive ouverte pluridisciplinaire HAL, est destinée au dépôt et à la diffusion de documents scientifiques de niveau recherche, publiés ou non, émanant des établissements d'enseignement et de recherche français ou étrangers, des laboratoires publics ou privés. 


\title{
Active joint visco-elasticity estimation of the human knee using FES
}

\author{
Seiya Sakaguchi, Gentiane Venture, Christine Azevedo and Mitsuhiro Hayashibe
}

\begin{abstract}
In order to understand the human motion control strategies and to restore these functions, or to artificially generate limbs motion it is necessary to have an accurate understanding of the limb dynamics. The inertial parameters can be identify easily, however the joint dynamics is still difficult to model due to the time change with muscle contraction level, fatigue and non-linear dynamics. Using Functional Electrical Stimulation (FES) we propose to identify the joint active dynamics with the pendulum test and to establish a relationship between the level of muscle contraction induced by the stimulation and the visco-elasticity. We measure the data of 2 healthy subjects and propose a model for the knee joint visco-elasticity changes.
\end{abstract}

\section{INTRODUCTION}

The viscoelastic properties of the human joints result in an extremely complex combination of elasticity, viscosity and friction of the joint constitutive elements and material deformation: the passive musculo-tendon, the connective tissues, the soft tissues and the contractive element of the muscles [1]. The stiffening of a joint is the result of complex changes that cannot be represented by the stiffening of muscles alone as it is accompanied by biomechanical changes of the surrounding tissues, stiffening of the tendons, as well as changes at the joint level such as the deposition of an extra amount of collagen according to [2].

When the muscles are not active (passive movements) it is possible and rather simple to observe the fundamental joint dynamics [3]. During such movements no contraction of muscles occurs around the concerned joint. The Wartenberg's test or "Pendulousness of the leg" [4], often used in medical studies of the knee is a typical passive motion of the kneejoint [5] [6]. However, during active movements (movements with contraction of muscles) the viscoelastic properties of the joints change with time, the level of muscle contraction, the external forces and fatigue [7]. Empirical models that describe the behavior of muscles during active movements are complex non-linear systems and numerically poorly referenced [8]. The redundancy of muscles actuating one joint is also a limitation to in-vivo studies. Using EMG to measure the level of activity of the muscle is sometimes used [9]. As controlling the level of voluntary contraction is not trivial, these model are not providing convincing

Authors acknowledge the support provided by the INRIA-JSPS joint program AYAME (2010-2012).

Gentiane Venture and Seiya Sakaguchi are with the Department of Mechanical Systems Engineering, Tokyo University of Agriculture and Technology, 2-24-16 Nakacho, Koganei, Tokyo, Japan venturedcc.tuat.ac.jp

Mitsuhiro Hayashibe and Christine Azevedo are with INRIA Sophia Antipolis-DEMAR Project and LIRMM, UMR5506 CNRS UM2, 161 Rue Ada, 34095 Montpellier, France hayashibed lirmm.fr results [10] [11] [12]. To overcome this issue, we propose in this paper to use Functional Electrical Stimulation (FES) to stimulate muscle and control the level of muscle contraction to identify the active knee joint visco-elastic properties using the "Pendulousness of the leg".

FES is one of the solutions to improve lost motor functions in persons with Spinal Cord Injury (SCI) or cerebral injury. The electrical currents artificially generate action potentials on the axons of the alpha motor neurons to induce muscle contraction in place of the Central Nervous System (CNS). The intended movement is achieved by the correct stimulation pattern, which depends on the specific applications and stimulation interface. The challenge in the present FES system starts with the problem of how to process the high nonlinearity and complexity of musculoskeletal systems, which complicate the model identification process. Most of the FES control research focused on its joint angle closed loop feedback [13]. However, some of the other works proposed to control joint stiffness to improve the stability of limbs. Matjacic and Bajd [14], [15] have demonstrated that a paraplegic subject, after appropriate training, is able to stabilize himself using his trunk muscles if a certain level of stiffness around the ankle joint is present. Jaime and Hunt [16], [17] have investigated the control of paraplegic ankle joint stiffness using FES while standing. They concluded that ankle stiffness control has the potential to ease the task of stabilizing upright posture by application of additional upperbody forces.

We first briefly detail the identification procedure, then we present our experimental protocol, finally the experimental results are detailed and analyzed.

\section{DYNAMICS MODELING OF THE KNEE JOINT AND IDENTIFICATION}

\section{A. Modeling human joints during passive movements}

The inverse dynamics of a multi-body system with $N$ degrees of freedom can be described by Eq. 1 [18]:

$$
\tau=\Gamma+Q=H\left(q, \dot{q}, \ddot{q}, I_{P}\right)+\tau^{v e f}
$$

where:

- $\tau$ is the $N \times 1$ vector of joint torques,

- $\Gamma$ is the $N \times 1$ vector of joint forces or torques due to the actuation: in the human body actuation is due to the contractions of antagonist muscles,

- $Q$ is the $N \times 1$ vector of generalized efforts representing the projection of the external forces and torques on the joint axes,

- $H$ is the $N \times 1$ vector of inertial, Coriolis, centrifugal and gravity forces, 
- $q$ is the $N \times 1$ vector of joint angle, $\dot{q}$ and $\ddot{q}$ are its first and second time derivatives,

- $I_{P}$ is the $(1 \times 10 N)$ vector of inertial parameters of the system: mass, inertia, first moment of inertia,

- $\tau^{v e f}$ is the $N \times 1$ vector of torque due to the viscoelasticity and friction of the joints. If the joint $i$ has no visco-elasticity $\tau_{i}^{v e f}=0$; if the joint $i$ is viscoelastic then $\tau_{i}^{v e f}=\varphi\left(q_{i}, \dot{q}_{i}\right)$.

During passive movement $\varphi(q, \dot{q})$ can be considered as a well-known model in biomechanics [5]. During passive movements a joint with viscoelastic properties has constant subject-specific parameters and can be represented by its stiffness $k$, its damping $h$ as shown in Eq. (2), where $q_{z}$ is the zero position. It has been shown in [3] that the friction coefficient is extremely small and does not need to be taken into account. This model provides a good description of the behavior for a medium range of motion (avoiding the boundaries); however it fails in describing the behavior at the boundaries as we have shown in [19]. In this paper we consider that the knee doesn't not reach its boundary in flexion, so it is enough to describe the joint behavior. Moreover, as we stimulate muscle at a fixed level of FES we can utilize the same model for each level of stimulation and calculate $k$ and $h$ for each stimulation level.

$$
\varphi(q, \dot{q})=k\left(q-q_{z}\right)+h \dot{q}
$$

The zero-position $q_{z}$ is defined as the normal resting position. It is to be noted that when unknown, the zero position can be identified as it appears in a linear form in the model as an offset. In our experiments, the zero position is the resting position when starting and finishing the measurements.

\section{B. Identification technique}

In order to compute the joint torque $\tau$ it is necessary to have accurate measurements of the geometric parameters, the inertial parameters $I_{P}$. These parameters are subjectspecific and vary considerably from one person to an other. The geometric parameters, mainly length of segments, are directly measured; inertial parameters cannot be measured and thus need to be estimated. A common method would be to interpolate those parameters from literature data [20]. Individual identification is also possible [21]. Once the parameters computed it is possible to identify the joint dynamics. The identification method we use was developed for robotics systems [22] and since then it has been applied widely to various mechanical systems [23], [24], [25]. It relies on the linear property of the inverse dynamics with respect to the parameters to be estimated.

The joint dynamics given by Eq. (2) has the joint dynamics parameters $k$ and $h$, in a linear form. Consequently the inverse dynamics (1) can be written as follows:

$$
\tau=D(q, \dot{q}, \ddot{q}) X
$$

- $X$ is the $(2 \times 1)$ vector of parameters to be estimated, $X=[\mathrm{k} \mathrm{h}]$,
- $D$ is the $(1 \times 2)$ regressor, function of the vector of joint angle $q$ and its first and second derivatives,

- $\tau$ is the joint torque and external forces and torques.

The dynamic model (3) is sampled along a movement. The $n_{S}$ samples give an over-determined linear system of equations:

$$
Y=W(q, \dot{q}, \ddot{q}) X+\rho
$$

where:

- $Y$ is the $\left(n_{S} \times 1\right)$ vector of joint torques, obtained by sampling $\tau$ :

- $W$ is the $\left(n_{S} \times 2\right)$ observation matrix (or regressor), obtained by sampling $D$ :

- $\rho$ is the $\left(n_{S} \times 1\right)$ vector of modeling and measurement errors.

After the computation of the minimal set of parameters that can be identified, named base parameters [26], which depend on the excitation properties of the movement used for identification, the solution $\hat{X}$ of Eq. (4) is obtained using the linear least squares method; which is implemented in many software packages with efficient algorithms. This method allows high flexibility, concatenation of different movements and computation of indicators for the interpretation of the results [18], [27], such as:

- the condition number of the regressor matrix $W$,

- the relative standard deviation $\sigma_{\hat{X}} \%$ for each of the identified parameters in $\hat{X}$.

\section{EXPERIMENTS}

Experiments were performed with the aim of identifying knee joint visco-elasticity for two healthy male athletes (Subject1 and Subject2) that were selected for this study. Priori to the experiments, the contents and purpose of the experiment was clearly explained to them and they gave a written consent.

The Inertial Measurement Units (IMU) is used as sensor in this experiment. The IMU has acceleration sensor and gyroscopes. The IMU used in this experiment measures the accelerations along 3-axis and the angular velocities around 3-axis. The IMU compute rotation angles around 3-axis from these measured values and reports measured values and computed values. We use the rotation angle and angular acceleration that we differentiate from the angular velocity to analyze the joint torque. The subject sits back in a high seat so that the feet do not touch the ground. The IMU is fixed at the subject's ankle on the face of the leg. We arrange the axes such that the gravity is along the vertical axis of the IMU. The set of experiment is shown on Fig. 1. We measure the distance between the IMU and the knee joint axis of flexion/extension. The operator extends the leg of subjects up to about 60 degrees, and releases it. We record the leg swings during these free motion. The leg stops naturally, then we repeat the operation. We records 3 swings of the leg in a row with the same condition. The subject has to relax the leg and not to contract his muscles. The leg swing is therefore a passive movement and not to touch something. 
TABLE I

THE IDENTIFIED JOINT VISCO-ELASTICITY PARAMETERS AT NO STIMULATION

\begin{tabular}{l|cc|cc|cc}
\hline \multicolumn{1}{l}{ Subject1 } \\
\hline parameter & $\hat{X}$ & $\sigma_{\hat{X}} \%$ & $\hat{X}$ & $\sigma_{\hat{X}} \%$ & $\hat{X}$ & $\sigma_{\hat{X}} \%$ \\
\hline$k[\mathrm{Nm} / \mathrm{rad}]$ & 0.941 & 0.93 & 0.874 & 0.99 & 0.994 & 1.05 \\
\hline$h[\mathrm{Nms} / \mathrm{rad}]$ & 0.048 & 3.65 & 0.047 & 3.73 & 0.052 & 4.18 \\
\hline Subject2 \\
\hline$k[\mathrm{Nm} / \mathrm{rad}]$ & 2.673 & 3.28 & 1.647 & 4.84 & 1.237 & 5.28 \\
\hline$h[\mathrm{Nms} / \mathrm{rad}]$ & 0.369 & 5.77 & 0.345 & 5.21 & 0.356 & 4.10 \\
\hline
\end{tabular}

We experiment with FES changing condition of stimulation strength. The muscle group is stimulated with amplitude modulation at a constant frequency $(30 \mathrm{~Hz})$ and constant PW (300 $\mu \mathrm{s}$ ), by a portable stimulator (Cefar physio 4, Cefar Medical, Lund, Sweden). We conduct the same pendulum test as above at different conditions of stimulation. As we are interested on the knee flexion-extension movement, the FES electrodes are set on the quadriceps of the subjects as Fig. 1. The quadriceps contracts during constant electrical current flow. First, we conduct experiment under the condition of no stimulation, in other words, stimulation current strength is $0 m A$, which correspond to totally passive movements. Next, we change the condition, the stimulation current strength is $9 m A$ for Subject1. We set the intensity of starting stimulation current according to subject's sensibility: we progressively raised current values until inducing stimulus feeling to the subjects. Such value was then chosen as starting stimulation current intensity. For Subject2, beginning current strength of stimulation is $12 \mathrm{~mA}$. The condition is changed progressively as the electric current of stimulation strength raised $1 \mathrm{~mA}$ at a time: $1.5 \mathrm{~mA}$ for Subject2. We conduct with again the test under no stimulation every two step of changing condition to relax muscle. We repeat this process, changing the condition until the stimulation current strength is $20 \mathrm{~mA}$, which is when the subject feels pain (Subject1). For Subject2, the maximal stimulation current strength is $28.5 \mathrm{~mA}$. At last, we experiment under the condition of no stimulation to verify the post-effect of stimulation. We estimate the knee joint visco-

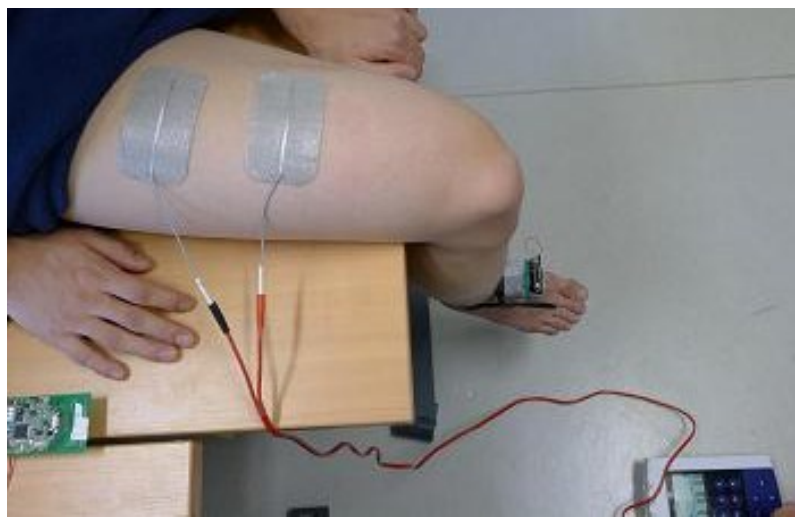

Fig. 1. Experimental installation: FES electrodes on the quadriceps, IMU sensor on the ankle

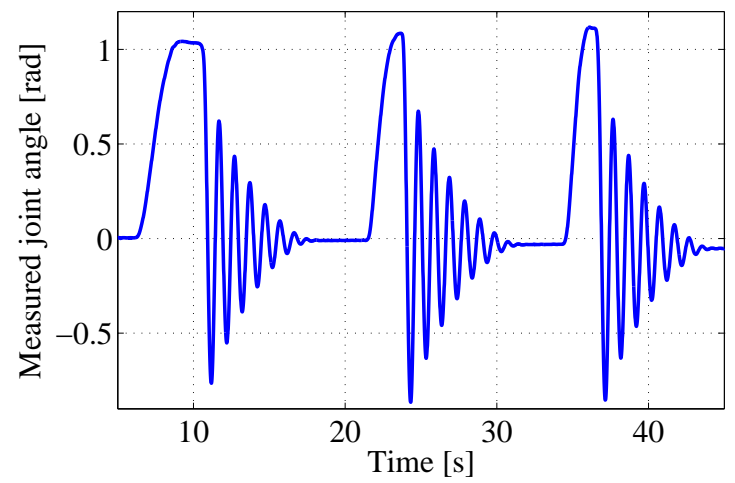

Fig. 2. Knee joint angle under condition of stimulation $0 m A, 17 m A, 18 m A$, $19 m A$ for Subject1

elasticity from the information of the IMU. We compute torque around the knee joint axis of flexion/extension with inverse dynamics from joint angular acceleration, angle and parameters of lower leg. We identify the joint visco-elasticity parameters as described in section II-B.

Fig. 2 shows the recorded angle around the knee joint axis at first condition of no stimulation for the Subject1 and Fig. 3 under different levels of stimulation. From these figures the effect of the stimulation, thus muscle contraction on the joint visco-elastic properties is clear. We extract sampling data that is part of the recorded full data, from the time the operator releases the subject's leg to the leg stop, and we identify the joint visco-elasticity parameters. For each stimulation level 3 swings are repeated, and we identify parameters at each swing. These parameters identified at each swing are summarized in Table I. The results of identification for Subject1 at a stimulation level of $19 \mathrm{~mA}$ are given in Table II. Similar results for all the stimulation level, and for Subject2 are obtained. Direct and cross validation to verify the validity of the estimated parameters are reported in Fig. 4 and Fig. 5, respectively (at no stimulation) and in Fig. 6 and Fig. 7, respectively (at 19mA stimulation). The summary of the identification results for each stimulation level are summarized on Fig. 8 for Subject1 and Fig. 9 for Subject2.

\section{RESULTS AND DISCUSSION}

The joint stiffness $k$ and the viscosity $h$ are estimated with good accuracy as seen from the low value of the relative standard deviations: $\sigma_{\hat{X} j} \%<6 \%$. There is a good correlation between the obtained values for each of the repeated tests. Fig. 4 and Fig. 5 give a graphical visualization of the validation. The joint torque obtained by computation of the inverse dynamics $Y$ (blue solid line) and the joint torque computed from the joint angle and estimated dynamics $W X$ (green dashed line) are compared. The error between the two $Y$-WX (black dotted line) is also given. Fig. 4 and gives the direct validation: the swing is in the data-set of identification. The error is equivalent to the vector of error in term of the least squares $\rho$. Fig. 5 gives cross validations: the swing is not in the data-set used for the identification. In 

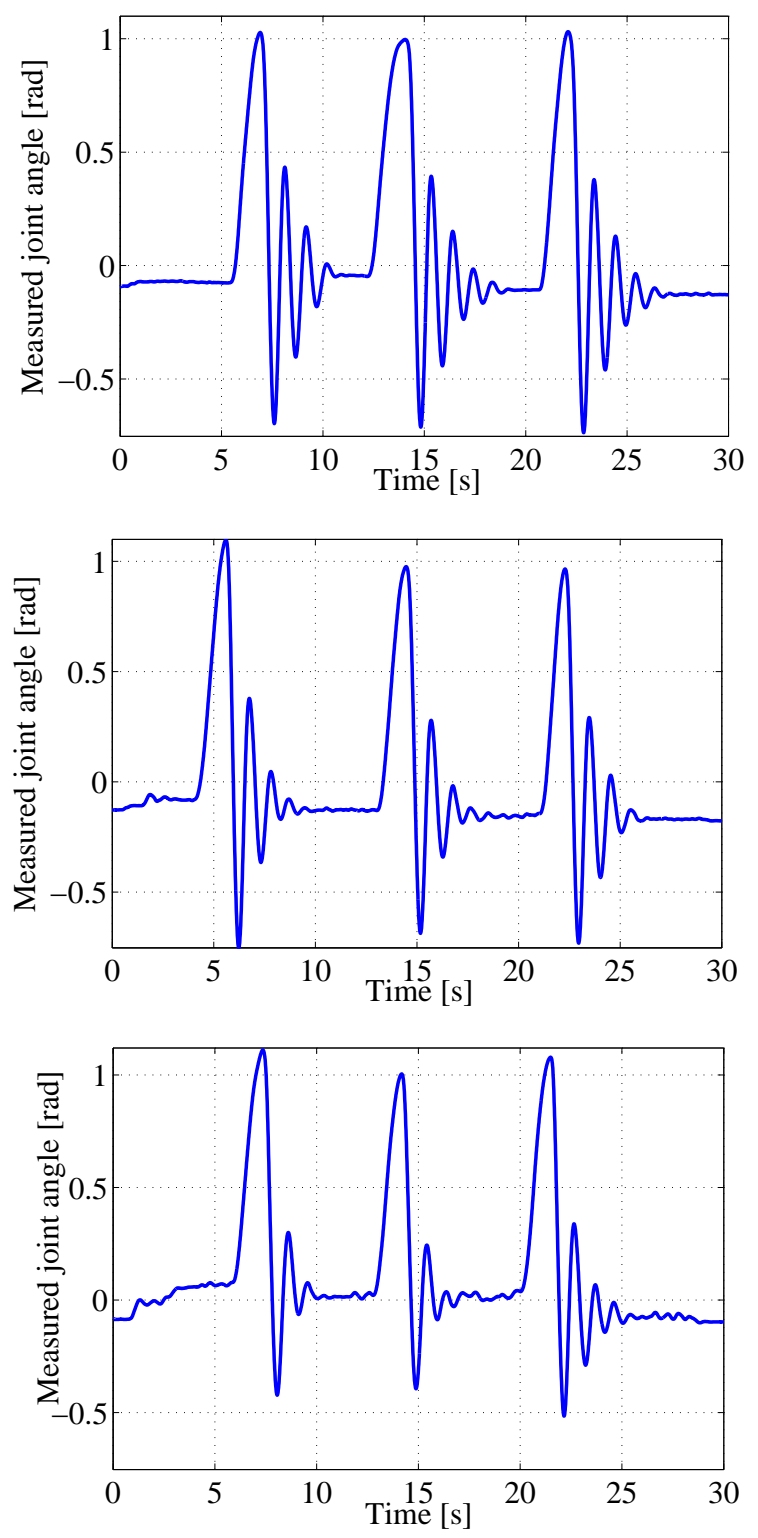

Fig. 3. Knee joint angle under condition of stimulation $17 \mathrm{~mA}, 18 \mathrm{~mA}, 19 \mathrm{~mA}$ for Subject1

TABLE II

THE IDENTIFIED JOINT VISCO-ELASTICITY PARAMETERS WITH STIMULATION

\begin{tabular}{|c|c|c|c|c|c|c|}
\hline \multicolumn{7}{|c|}{ Subject1 (19n } \\
\hline parameter & $\hat{X}$ & $\sigma_{\hat{X}} \%$ & $\hat{X}$ & $\sigma_{\hat{X}} \%$ & $\hat{X}$ & $\sigma_{\hat{X}} \%$ \\
\hline$k[\mathrm{Nm} / \mathrm{rad}]$ & 1.502 & 1.96 & 1.649 & 1.73 & 1.354 & 2.75 \\
\hline$h[\mathrm{Nms} / \mathrm{rad}]$ & 0.198 & 3.07 & 0.197 & 3.12 & 0.180 & 4.38 \\
\hline \multicolumn{7}{|c|}{ Subject2 (27mA of stimulation) } \\
\hline$k[\mathrm{Nm} / \mathrm{rad}]$ & 3.525 & 5.52 & 4.724 & 3.85 & 3.634 & 4.75 \\
\hline$h[\mathrm{Nms} / \mathrm{rad}]$ & 1.436 & 4.89 & 1.175 & 5.92 & 1.009 & 4.98 \\
\hline
\end{tabular}

Fig. 5, the motion is the third swing, and $X$ is the vector of estimated parameters during the second swing. The error is low, showing good estimation of the joint torque using the passive model with the identified parameters.
The identification results for all swings under each condition present the same properties. The identified parameters at $19 \mathrm{~mA}$ stimulation are summarized in Table II. The direct and cross validations at condition that stimulation current strength is $19 \mathrm{~mA}$ given in Fig. 6 and Fig. 7 are quite similar to the results of the pure passive movement $(0 \mathrm{~mA})$. Only a slight degradation of the torque reconstruction can be seen. The 2parameter linear model describes fairly well the behavior of the knee joint under FES induced muscle contractions.

Fig. 8 shows the identified parameters of stiffness $k$ and viscosity $h$ at each condition of stimulation intensity for Subject1. In these figures, the electrical current stimulation is the horizontal axis, and the identified parameters are plotted vertically. Similarly, the identified parameters for the Subject 2 are shown on Fig. 9. These figures show that the joint visco-elasticity varies according to the stimulation current strength. The results for these subjects, the relationship between strength of FES and the joint visco-elasticity can be

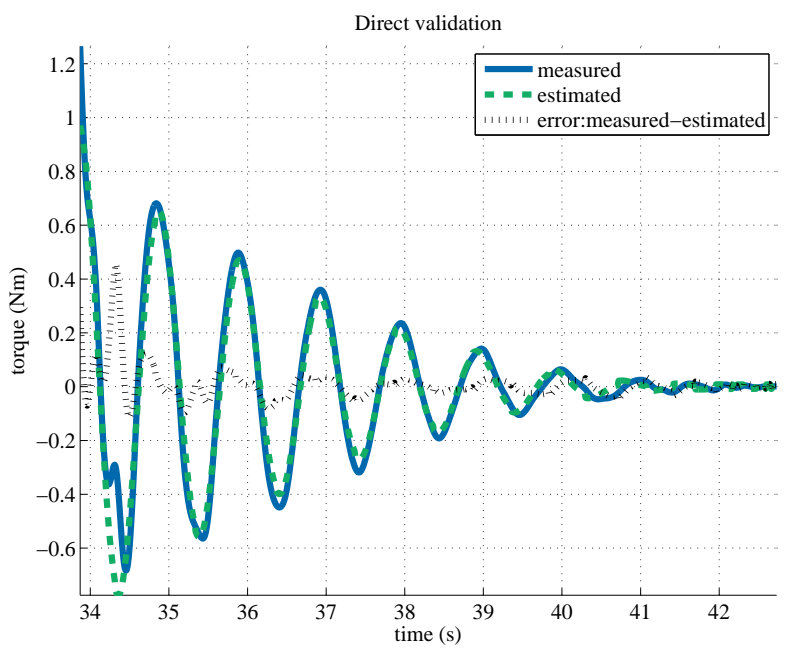

Fig. 4. Direct validation at no stimulation for Subject1

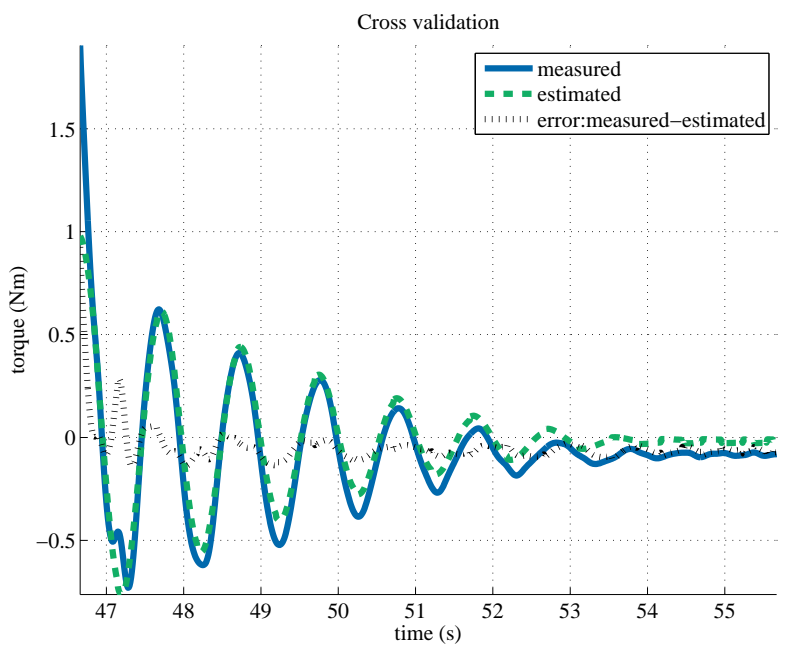

Fig. 5. Cross validation at no stimulastion for Subject1 
clearly evaluated. Our protocol also allows to verify that the identified parameters are not affected by fatigue at all time of no stimulation.

We now look at the variations of the identified joint viscoelasticity parameters versus stimulation current strength. For both subjects the change in $k$ and $h$ with respect to stimulation present the same profile, despite a variation in the relative values. The stiffness $k$ is unchanged before a certain threshold (of $14 \mathrm{~mA}$ for the Subject1, and 19.5mA for Subject2) and then linearly increasing over this stimulation threshold. The plotted data of the stiffness $k$ can be approximated by a function of Eq. 5. While the viscosity $h$ follow an exponential increase and can be modeled by Eq. 6 .

$$
\begin{array}{r}
k(\text { stim })= \begin{cases}k_{s} & (\text { stim } \leqq 14) \\
k_{1} \text { stim }-k_{2} & (\text { stim }>14)\end{cases} \\
h(\text { stim })=h_{1}+h_{2} \times \exp \left(h_{e} \times \text { stim }\right)
\end{array}
$$

where: stim is the stimulation current strength.

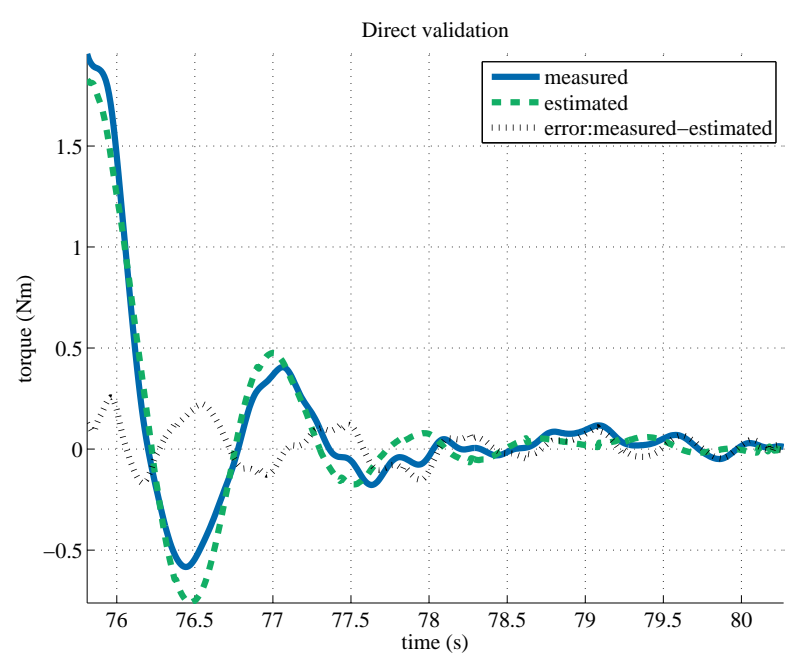

Fig. 6. Direct validation at stimulation of $19 \mathrm{~mA}$ for Subject1

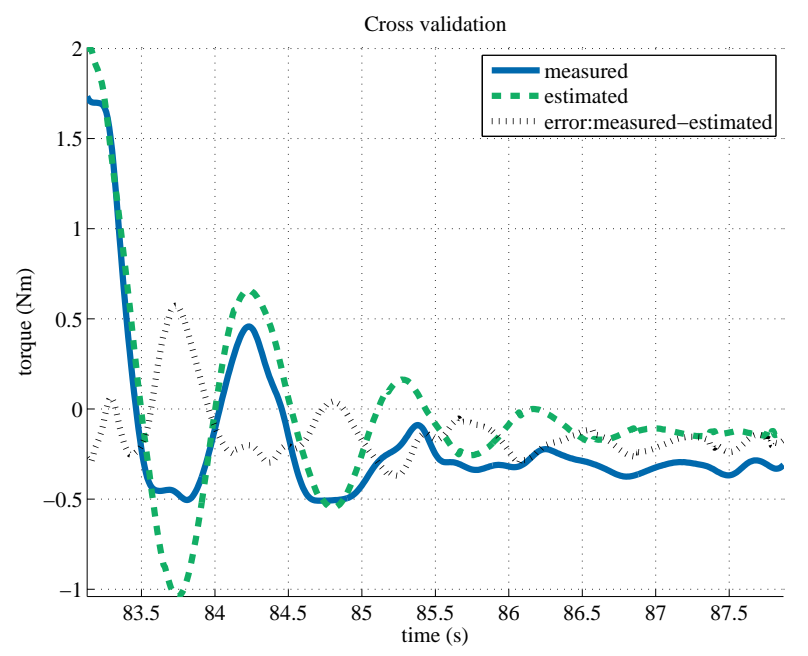

Fig. 7. Cross validation at stimulation of $19 \mathrm{~mA}$ for Subject1
We identify the parameters $k_{s}, k_{1}, k_{2}, h_{1}, h_{2}, h_{e}$ for each candidate. The numerical results are given by Eq. 7, Eq. 8 for Subject1, and by Eq. 9 and Eq. 10 for Subject2.

$$
\begin{aligned}
& k(\text { stim })= \begin{cases}0.915 & (\text { stim } \leqq 14) \\
0.117 \text { stim }-0.723 & (\text { stim }>14)\end{cases} \\
& h(\text { stim })=0.0450+1.82 \times 10^{-6} \times \exp (\operatorname{stim} \times 0.597) \\
& k(\text { stim })= \begin{cases}1.21 & (\text { stim } \leqq 19.5) \\
0.367 \text { stim }-5.94 & (\text { stim }>19.5)\end{cases} \\
& h(\text { stim })=0.280+1.12 \times 10^{-4} \times \exp (\operatorname{stim} \times 0.334)
\end{aligned}
$$

These equation results are shown by the black line in Fig. 8 and Fig. 9 by the black line. One can note that at the maximal stimulation, the stiffness drops dramatically for both subject to a level that is below to passive movement, though the viscosity $h$ continues to increase according to the exponential. It provides an interesting insight about the muscle behavior.

\section{CONCLUSION}

Using FES on the quadriceps and the pendulum test of the leg we identify the knee joint visco-elasticity under different conditions of muscle contraction. The results show that the pendulum test provides the necessary information to model the knee-joint changes with respect to muscle contraction induces by FES. The joint visco-elasticity under a constant contraction can be measured and is modeled by a 2-parameter
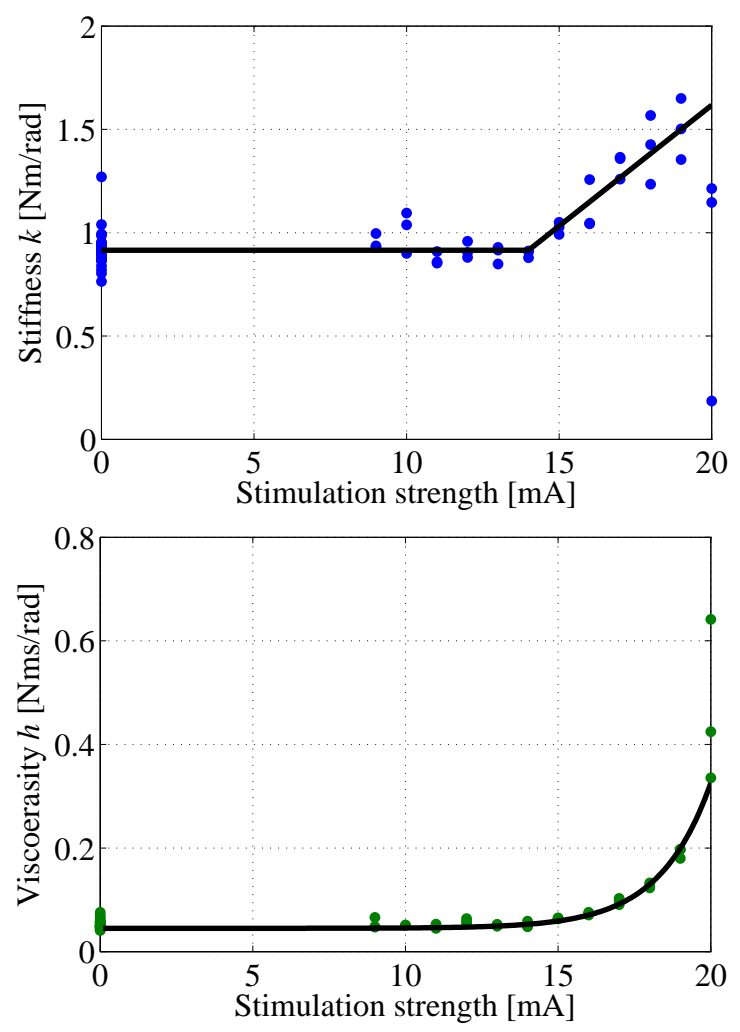

Fig. 8. Identified parameters for the Subject1 
linear model. Moreover, the changes in the visco-elasticity with respect to muscle contraction follow a linear profile for $k$ and an exponential variation for $h$. At the maximal supported contraction level the elasticity drops significantly. This results are obtained for two athletes. The low level of body fat and the high response of the muscle provide an almost noise-free response. And we believe they can be extended to non-athletes. In particular for FES implanted patients, these results are of importance as they allow to develop novel controller to assist gait. These results also provide insight about the global joint behavior under contraction, which is crucial to understand human complex movement control, and active joint stiffness and viscosity control. We have studied the relation between stimulation-stiffness and damping this time, to have more physiological relationship, we could consider in the future to use directly the stimulation parameters as activation input, though the relation between activation and stimulation is yet to be defined.

\section{REFERENCES}

[1] R.J. Johns and V. Wright, "Relative importance of various tissues in joint stiffness," J. Appl. Physiol., vol. 17, no. 5, pp. 824-828, 1962.

[2] A.F. Thilmann, S.J. Fellows, and H.F. Ross, "Biomechanical changes at the ankle joint after stroke," J. of Neurology, Neurosurgery, and Psychiatry, vol. 54, pp. 134-139, 1991.

[3] G. Venture, K. Yamane, T. Yamamoto, and Y. Nakamura, "Identification of human limb visco-elasticity using robotics methods to support the diagnosis of neuromuscular diseases," Int. J. of Robotic Research, vol. 28, pp. 1322-1333, 2009.

[4] R. Wartenberg, "Pendulousness of the leg as a diagnostic test," Neurology, vol. 1, pp. 18-24, 1951.
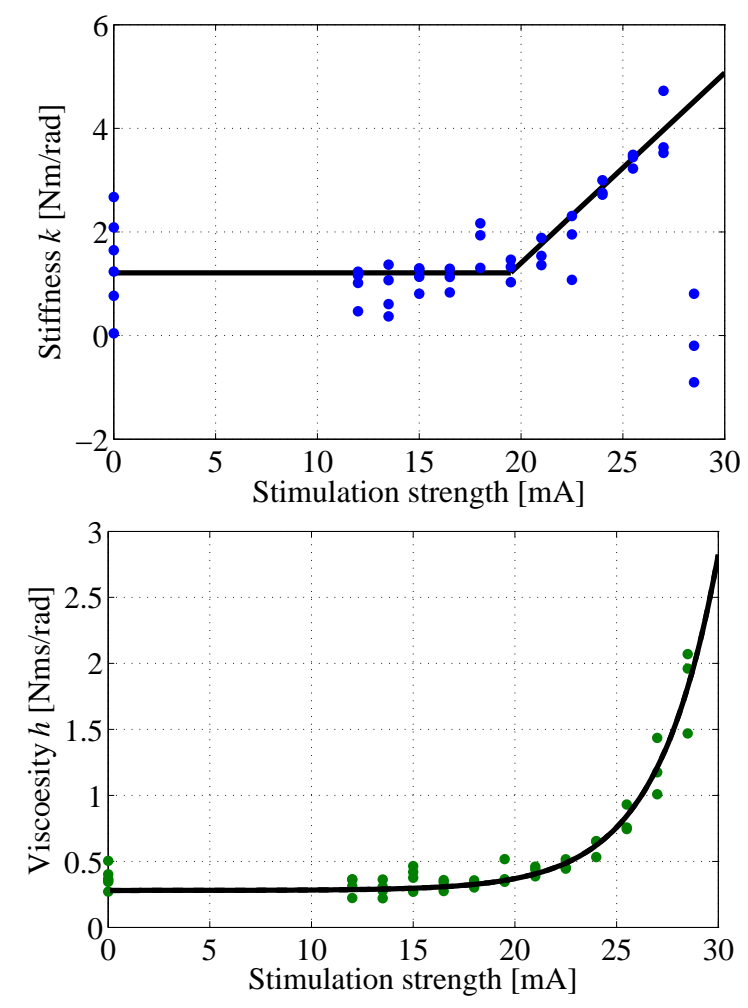

Fig. 9. Identified parameters for the Subject2
[5] A.C. Oatis, "The use of a mechanical model to describe the stiffness and damping characteristics of the knee joint in healthy adults," Phys. Ther., vol. 73, no. 11, pp. 740-749, 1993.

[6] C.H. Such, A. Unsworth, V. Wright, and D. Dowson, "Quantitative study of stiffness in the knee joint," Annals of the Rheumatic Diseases, vol. 34, pp. 286-291, 1975.

[7] A. Prochazka, D.J. Bennett, M.J. Stephens, R. Sears-Duru, T. Roberts, and J.H. Jhamandas, "Measurement of rigidity in parkinson's disease," Movement Disorders, vol. 12, pp. 24-32, 1997.

[8] G Venture, K Yamane, and Y Nakamura, "Application of non-linear least square method to estimate the muscle dynamics of the elbow joint," in IFAC - Int. Conf. on System Identification, pp. 1168-1173, 2006.

[9] R. Osu and H. Gomi, "Multijoint muscle regulation mechanisms examined by measured human arm stiffness and emg signals," J. of Neurophysiology, vol. 81, no. 4, pp. 1458-1468, 1999.

[10] K. Amankwah, R.J. Triolo, and R. Kirsch, "Effects of spinal cord injury on lower-limb passive joint moments revealed through a nonlinear viscoelastic model," J. of Rehabilitation Research and Development, vol. 41, no. 1, pp. 15-32, January-February 2004.

[11] Y. Xu and J.M. Hollerbach, "A robust ensemble data method for identification of human joint mechanical properties during movement," IEEE Trans. on Biomedical Eng., vol. 46, pp. 409-419, 1999.

[12] H.I. Giesbrecht, M. Baker, D. Ludvig, R. Wagner, and R.E. Kearney, "Identification of time-varying intrinsic and reflex joint stiffness," in Proc. of the 28th IEEE EMBS Annual Int. Conf., New York City, USA, pp. 288-291, 2006.

[13] M. Ferrarin, F. Palazzo, R. Riener, and J. Quintern, "Modelbased control of FES induced single joint movements", IEEE Transactions on Neural Systems and Rehabilitation Engineering, 9(3), pp.245-257, 2001.

[14] Z. Matjacic and T. Bajd, "Arm-free paraplegic standing-Part I: control model synthesis and simulation", IEEE Trans. Rehabil. Eng., vol.6, pp.125-138, 1998.

[15] Z. Matjacic and T. Bajd, "Arm-free paraplegic standing?part II: experimental results", IEEE Trans. Rehabil. Eng., vol.6, pp.139-150, 1998.

[16] Ralf-Peter Jaime, Z. Matjacic, and T. Bajd, "Paraplegic standing supported by FES-controlled ankle stiffness", IEEE Trans. Neural Sys. Rehab. Eng., vol.10, pp. 239-248, 2002.

[17] Henrik Gollee, Ken J. Hunt, and Duncan E. Wood, "New Results in Feedback Control of Unsupported Standing in Paraplegia", IEEE Trans. Neural Sys. Rehab. Eng., vol.12, pp. 73-80, 2004.

[18] W. Khalil and E. Dombre, Modeling, identification and control of robots, Hermès Penton, London-U.K, 2002.

[19] G. Venture, K. Yamane, Y. Nakamura, and M. Hirashima, "Estimating viscoelastic properties of human limb joints based on motion capture and robotic identification technologies," in Proc. of the IEEE Int. Conf. on Intelligent Systems and Robots, pp. 624-629, 2007.

[20] R. Dumas, L. Cheze, and J.-P. Verriest, "Adjustments to mcconville et al. and young et al. body segment inertial parameters," J. Biomechanics, vol. 40, pp. 543-553, 2007.

[21] K. Ayusawa, G. Venture, and Y. Nakamura, "Identification of humanoid robots dynamics using floating-base motion dynamics," in Proc. of the IEEE Int. Conf. on Intelligent Systems and Robots, pp. 2854-2859, 2008.

[22] M. Khalil, W. Gautier and J.F. Kleinfinger, "Automatic generation of identification models of robots," Int. J. of Robotics and Automation, vol. 1, no. 1, pp. 2-6, 1986.

[23] P. Aarnio, K. Koskinen, and S. Salmi, "Simulation of the hybtor robot," in Proc. of the 3rd Int. Conf. on Climbing and Walking Robots, pp. 267-274, 2000.

[24] M.W. Hannan and I.D. Walker, "Kinematics and the implementation of an elephant's trunk manipulator and other continuum style robots," J. of Robotic Systems, vol. 20, no. 2, pp. 45-63, 2003.

[25] G. Venture, K. Yamane, and Y. Nakamura, "In-vivo estimation of the human elbow joint dynamics during passive movements using musculo-skeletal model computations," in Proc. of the IEEE/RASEMBS Int. Conf. on Biomedical Robotics and Biomechatronics, pp. 205-210, 2006.

[26] M. Gautier, "Numerical calculation of the base inertial parameters," J. of Robotic Systems, vol. 8(4), pp. 485-506, 1991.

[27] M. Gautier and W. Khalil, "Exciting trajectories for the identification of base inertial parameters of robots," Int. J. of Robotic Research, vol. 11, no. 4, pp. 363-375, 1992. 